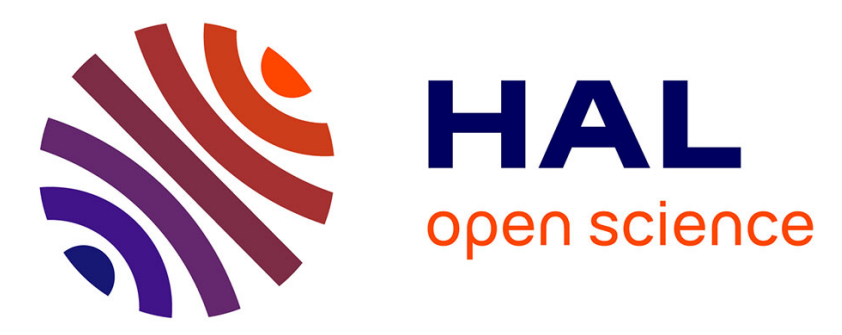

\title{
Frequency-doubling effect in acoustic reflection by a nonlinear, architected rotating-square metasurface
} Xinxin Guo, Vitali Goussev, Vincent Tournat, Bolei Deng, Katia Bertoldi

\section{To cite this version:}

Xinxin Guo, Vitali Goussev, Vincent Tournat, Bolei Deng, Katia Bertoldi. Frequency-doubling effect in acoustic reflection by a nonlinear, architected rotating-square metasurface. Physical Review E , 2019, 99 (5), 10.1103/PhysRevE.99.052209 . hal-02132143

\section{HAL Id: hal-02132143 \\ https://hal.science/hal-02132143}

Submitted on 16 May 2019

HAL is a multi-disciplinary open access archive for the deposit and dissemination of scientific research documents, whether they are published or not. The documents may come from teaching and research institutions in France or abroad, or from public or private research centers.
L'archive ouverte pluridisciplinaire HAL, est destinée au dépôt et à la diffusion de documents scientifiques de niveau recherche, publiés ou non, émanant des établissements d'enseignement et de recherche français ou étrangers, des laboratoires publics ou privés. 


\title{
Frequency-doubling effect in acoustic reflection by a nonlinear, architected rotating-square metasurface
}

\author{
Xinxin Guo,* Vitalyi E. Gusev, and Vincent Tournat ${ }^{\dagger}$ \\ LAUM, CNRS UMR 6613, Le Mans Université, Av. O. Messiaen, 72085 Le Mans, France \\ Bolei Deng and Katia Bertoldi \\ John A. Paulson School of Engineering and Applied Sciences, \\ Harvard University, Cambridge, 02138 MA, USA
}

(Dated: May 16, 2019)

\begin{abstract}
Nonlinear acoustic metamaterials offer the potential to enhance wave control opportunities beyond those already demonstrated via dispersion engineering in linear metamaterials. Managing the nonlinearities of a dynamic elastic system however remains a challenge, and the need now exists for new strategies to model and design these wave nonlinearities. Inspired by recent research on soft architected rotating-square structures, we propose herein a design for a nonlinear elastic metasurface with the capability to achieve nonlinear acoustic wave reflection control. The designed metasurface is composed of a single layer of rotating squares connected to thin and highly deformable ligaments placed between a rigid plate and a wall. It is shown that during the process of reflection at normal incidence, most of the incoming fundamental wave energy can be converted into the second harmonic wave. A conversion coefficient of approximately 0.8 towards the second harmonic is derived with a reflection coefficient of $<0.05$ at the incoming fundamental frequency. The theoretical results obtained using the harmonic balance method (HBM) for a monochromatic pump source are confirmed by time-domain simulations for wave packets. The reported design of a nonlinear acoustic metasurface can be extended to a large family of architected structures, thus opening new avenues for realistic metasurface designs that provide for nonlinear or amplitude-dependent wave tailoring.
\end{abstract}

\section{INTRODUCTION}

Acoustic metamaterials composed of local resonators have proven to be of great interest, due to their ability to perform a variety of wave control functionalities at wavelengths much longer than the dimensions of the resonant elements. A wide array of novel acoustic phenomena such as slow sound [1-3], negative refraction [410], subwavelength wave guiding [11, 12], sound absorption [13-20] and cloaking [21-24] have been demonstrated in appropriately designed metamaterials. Compared to the metamaterials composed of linear resonators, nonlinear metamaterials offer a rich and diverse set of nontrivial acoustic phenomena, including asymmetric transmission [11, 25-28], nonlinear pulse and soliton propagation [29-31], harmonic generation [32, 33] and breathers $[34,35]$. Nevertheless, the design of nonlinear metamaterials, which was initially investigated in optics for the purpose of enhancing the higher harmonic generation [36-38], has been studied much less extensively in the acoustic field [39].

The key limitations in developing nonlinear acoustic metamaterials pertain to the typically weak efficiency of their nonlinear response, combined with a lack of control over this nonlinearity. Examples of tailoring the acoustic or elastic wave nonlinearity of a system are found in granular crystals, yet the tunability is intrinsically limited due to the Hertz-Mindlin contact behavior [40, 41].

\footnotetext{
* guoxinxin910118@gmail.com

$\dagger$ vincent.tournat@univ-lemans.fr
}

Being able to manage the wave nonlinearity of a system, over a wider parameter space, thus appears as the main challenge to developing nonlinear acoustic metamaterials.

In studying a lumped-element model of a nonlinear metasurface [42], we recently demonstrated that nonlinear acoustic effects can be enhanced in a subwavelength metasurface comprising nonlinear oscillators, thanks to the resonance process. This process intrinsically increases the characteristic interaction times as well as local wave amplitudes. We have reported a nonlinear frequency conversion effect from the incoming fundamental wave to the reflected second harmonic. However, the key link between the lumped-element model of this nonlinear metasurface and a realistic structure is missing. More specifically, the method of designing elastic springs with an effective quadratic nonlinearity still needs to be determined.

Recent research has demonstrated that soft architected materials enable manipulating and controlling elastic and acoustic waves [31, 43-48]. The intrinsic structure and property of this class of architected materials are not only modifiable by harnessing the elastic buckling resulting from different statically-produced pre-deformations $[44,46,47]$, but also dynamically tunable over a broad range of frequencies by taking advantage of geometric nonlinearities in the basic building blocks [31, 48]. As such, these nonlinearities provide the opportunity to expand the ability of existing metamaterials and enable them to support a wide variety of dispersive and nonlinear wave propagation.

Inspired by the latest research on the dynamics of soft 
architected materials comprising rotating units [31, 49], our attention has been drawn to the fact that the local rotational degree of freedom necessarily leads to the presence of sinusoidal functions of the angle of rotation in the motion equations. These nonlinear functions of wave variables constitute geometric type sources of wave nonlinearity and are found to depend on the building blocks (elasticity, geometry, inertia) of the architected structure. Consequently, in the aim of proposing a realistic design of a nonlinear elastic metasurface that accomplishes the same nonlinear conversion as in [42] but with a higher efficiency and over a much larger parameter space, the present paper analyzes a metasurface composed of a single layer of rotating units that are connected with a rigid moving plate and a wall, via thin and highly deformable ligaments. Special focus is placed on the nonlinear reflection process, thus leading to an optimal conversion from an incident sinusoidal wave towards its reflected second harmonic. By adjusting the physical properties of the metasurface, the desired nonlinear conversion is demonstrated to be feasible over a wide parameter space, hence enabling the extension of the proposed single design to a family of dynamic rotating-element metastructures. The predictive theoretical framework developed is also expected to help manage the wave nonlinearity by metamaterials and moreover guide future experiments in this field.

\section{NONLINEAR ELASTIC METASURFACE DESIGN AND THE REFLECTION PROBLEM AT NORMAL INCIDENCE}

In the previous research on wave propagation in a soft architected material made of rotating square units [31], it has been demonstrated that the studied 3D-printed material can be efficiently described by a lump elastic model. Accordingly, based on the theoretical model employed in [31], we propose herein a realistic design for a nonlinear metasurface. Note that the spring stiffness can be tuned by printing ligaments with different geometrical parameters or geometries, or by changing the printed material, in principle. As shown in Fig. 1, this design comprises periodically arranged rigid squares, connected via ligaments at their corners to a moving rigid plate (at the front end of the single square layer) and a fixed wall (at the back end of this square layer). The moving plate is assumed to be non-deformable and thus its possible vibration modes are not considered here. The ligaments are considered to be thin, massless and highly deformable, thus playing the role of elastic springs. The metasurface unit cell is composed of two identical squares with elementary masses $m$ sandwiched between the solid plate with a surface mass density of $2 m_{0}$ and the rigid wall. The two unit cell squares, featuring the same initial angle of rotation $\theta_{0}$ as defined in Fig. 1, are placed in symmetrical positions at rest. Since a horizontal force applied to the plate produces simultaneously square trans-

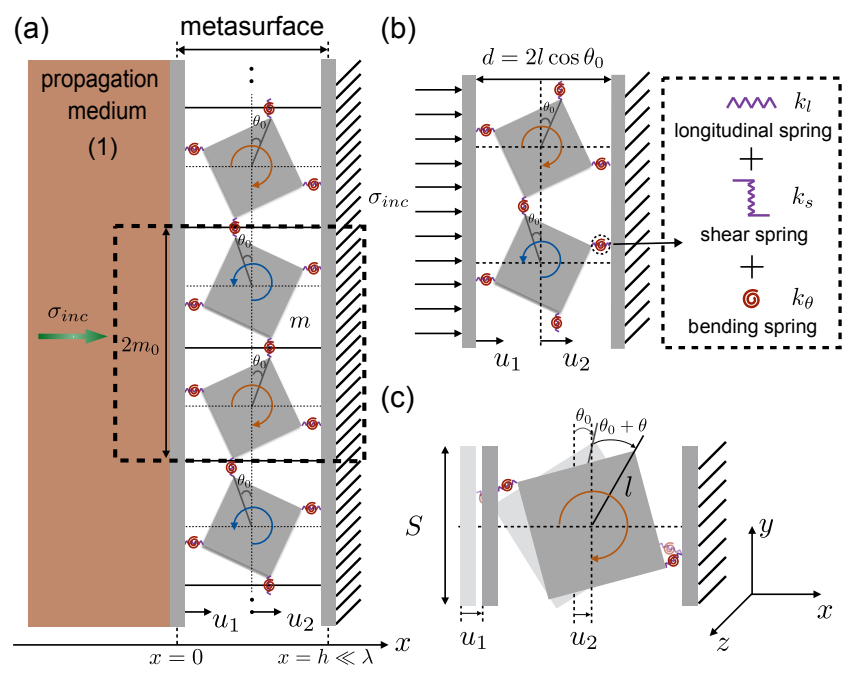

Figure 1. Nonlinear metasurface design: (a) Single layer of periodically arranged rigid squares sandwiched between a moving rigid plate and a fixed wall, with elastic springs running between all the elements. The propagation medium (1) in front of the designed metasurface is assumed to be semiinfinite. (b) The metasurface unit cell is composed of two identical squares with elementary mass $m$. The front rigid plate has an elementary mass $2 m_{0}$. (c) Due to symmetry, taking into account the motion of just one square of mass $m$ and the face plate with a mass per unit length of $m_{0}$ is demonstrated to be sufficient for the considered reflection problem at normal incidence.

lation and rotation, we take into account three different springs at each square vertex, to simulate the elastic behaviors of the ligaments : a longitudinal (compression or tension) spring with stiffness $k_{l}$, a shear spring with stiffness $k_{s}$, and a bending spring with stiffness $k_{\theta}$. During the structure deformation, the connections elements are maintained, as assumed in the work of Deng et al. [31].

The proposed metasurface structure is assumed to be infinitely long along the vertical direction $y$, while the plate thickness along $x$ is assumed to be significantly less than that of the single square layer. The considered design then is a $2 \mathrm{D}$ metastructure in the $(x, y)$ plane; moreover, the elementary lateral surface area of the metasurface unit cell is denoted by $S$.

Throughout this paper, focus is placed on the reflection process by the designed metasurface, with the propagation medium (1) in front of the metasurface assumed to be semi-infinite. More specifically, in order to reduce the complexity of the considered nonlinear problem, only the normal incidence is taken into account in our study. The metasurface width $h$ along the $x$ direction is assumed to be much smaller than the acoustic wavelength in medium (1), i.e., $h \ll \lambda$. Let's now consider a plane stress wave $\sigma_{\text {inc }}$ of amplitude $A_{\text {inc }}$ incident from $-\infty$ and propagating along the positive $x$ direction. The problem therefore is one-dimensional, and the incident and reflected waves can be written as a function of $x-c t$ and $x+c t$, respectively (using the time convention $i \omega t$ ), with $c$ de- 
noting wave velocity in the propagation medium. The total stress $\sigma$ can be decomposed into an incoming stress wave and a reflected stress wave $\sigma=\sigma_{i n c}+\sigma_{\text {ref }}$. The one-dimensional wave equation,

$$
\rho \frac{\partial^{2} u_{x}}{\partial t^{2}}=\frac{\partial \sigma}{\partial x}
$$

with $\rho$ as the mass density of the propagation medium and $u_{x}$ the displacement along the $x$ direction, must be satisfied everywhere and especially on the metasurface at $x=0$, which leads to the following relationship between the incident and reflected waves for the considered problem:

$$
\sigma_{r e f}=\sigma_{i n c}+\rho c \frac{\partial u_{1}}{\partial t}
$$

where $u_{1}$ denotes the displacement of the plate with a surface mass density of $m_{0}$.

Since the single square layer is periodically arranged and assumed to be infinitely long, with homogenous excitation along $y$, the two squares of each unit cell translate with the same displacement and moreover rotate with the same dynamic angle yet in opposite directions. Consequently, the motions of just one square and of its face plate are sufficient to describe the full dynamics.
For a systematic analysis, we introduce the following dimensionless parameters: normalized displacements $U_{i}=u_{i} / 2 l,(\mathrm{i}=1,2)$ of the plate and squares, respectively, with $2 l$ denoting the diagonal length of the squares, pulsation $\Omega=\omega / \omega_{0}$ with $\omega_{0}=\sqrt{k_{l} / m}$, time $\tau=\omega_{0} t$, inertial moment of squares $\alpha=J / m l^{2}$, normalized shear, bending stiffnesses $K_{s}=k_{s} / k_{l}$ and $K_{\theta}=k_{\theta} / k_{l} l^{2}$ respectively, and lastly mass ratio $\alpha_{m}=m_{0} / m$.

Based on previous results and validations [31, 49], the springs are assumed to behave linearly and dissipation is accounted for via linear viscous damping associated with the respective translation and rotation motions of each square. The characteristic dissipation parameters $\Gamma_{u}$ and $\Gamma_{\theta}$ are normalized as $\eta_{u}=\Gamma_{u} / m \omega_{0}$ and $\eta_{\theta}=\Gamma_{\theta} / m \omega_{0}$ for the translation and rotation, respectively. In Ref. [42], the effects of different levels of metasurface intrinsic dissipation have been studied: a stronger dissipation requires larger excitation amplitudes to achieve the same quantitative nonlinear effects in reflection. In the present work, it is considered that the dissipation remains relatively weak with a dimensionless value of $\eta_{u}=\eta_{\theta}=0.001$. Thus, for each square and the front plate occupying a lateral surface area $S$ in the $(y, z)$ plane, the governing motion equations are written as:

$$
\left\{\begin{aligned}
\alpha_{m} \frac{\partial^{2} U_{1}}{\partial \tau^{2}}= & -2 f_{i n c}(\Omega, \tau)-\gamma \frac{\partial U_{1}}{\partial \tau}-U_{1}+U_{2}-\eta_{u} \frac{\partial U_{1}}{\partial \tau}+\eta_{u} \frac{\partial U_{2}}{\partial \tau}+\frac{1}{2}\left(\cos \left(\theta_{0}\right)-\cos \left(\theta_{0}+\theta\right)+\eta_{\theta} \sin \theta_{0} \frac{\partial \theta}{\partial \tau}\right) \\
\frac{\partial^{2} U_{2}}{\partial \tau^{2}}= & U_{1}-2 U_{2}+\eta_{u} \frac{\partial U_{1}}{\partial \tau}-2 \eta_{u} \frac{\partial U_{2}}{\partial \tau} \\
\alpha \frac{\partial^{2} \theta}{\partial \tau^{2}}= & -6 K_{\theta}\left(\theta+\eta_{\theta} \frac{\partial \theta}{\partial \tau}\right)+2\left(U_{1}+\eta_{u} \frac{\partial U_{1}}{\partial \tau}\right) \sin \left(\theta_{0}+\theta\right)+6 \sin \left(\theta_{0}+\theta\right)\left(\cos \left(\theta_{0}+\theta\right)-\cos \left(\theta_{0}\right)-\eta_{\theta} \sin \theta_{0} \frac{\partial \theta}{\partial \tau}\right) \\
& -2 K_{s} \cos \left(\theta_{0}+\theta\right)\left(\sin \left(\theta_{0}+\theta\right)-\sin \left(\theta_{0}\right)+\eta_{\theta} \cos \theta_{0} \frac{\partial \theta}{\partial \tau}\right)
\end{aligned}\right.
$$

where $f_{\text {inc }}(\Omega, \tau)=\sigma_{\text {inc }}(\Omega, \tau) S / 2 k_{l} l$ denotes the normalized force applied to the plate due to the incident stress wave, $\gamma=\rho c S / m \omega_{0}$ the dimensionless impedance parameter representing the ratio of the propagation medium (1) impedance to the mechanical impedance of the metasurface.

When a normal incident stress wave interacts with the metasurface, and under the condition that the squares are initially rotated at nonzero angles $\theta_{0}$, the translation of the front plate induces both translation and rotation of the squares, along the $x$ direction and around the $z$ direction, respectively. Under a linear assumption for all springs, the elastic forces applied to each metasurface element are proportional to the spring elongations. However, since the square units rotate, the geometric nonlinearity of the structure is activated due to the sinusoidal dependence of spring deformations on the angle of rotation of the squares, as shown in Eq. (3). Interestingly, as a consequence of this geometric nonlinearity dependent on structural design, it can be tuned along with the linear elastic properties in order to produce specific nonlinear wave effects.

In the presence of nonlinearity, the reflected wave spectrum from a monochromatic incident wave at frequency $\omega$ may contain harmonics of the incident wave. Consequently, it is assumed that at the boundary $x=0$, the reflected wave is composed of harmonics with the complex amplitude $\widetilde{R}_{n}(1 \leq n \leq N)$ relative to the incident wave amplitude, denoted by $\widetilde{A}_{i n c}$. In the following discussion and for the sake of simplicity, $\widetilde{R}_{n}$ and $R_{n}$ will be used to represent the complex reflection coefficient of the $\mathrm{n}$-th harmonic and its magnitude, respectively. 


\section{ANALYSIS OF THE LINEARIZED METASURFACE: PARAMETER DEFINITIONS AND FREQUENCY RESPONSE}

In the linear and weakly dissipative configuration, i.e. with fixed dissipation parameters $\eta_{u}=\eta_{\theta}=0.001$ and a linear approximation of trigonometric functions as $\cos \left(\theta_{0}+\theta\right) \approx \cos \theta_{0}-\sin \left(\theta_{0}\right) \theta$ and $\sin \left(\theta_{0}+\theta\right) \approx$ $\sin \theta_{0}+\cos \left(\theta_{0}\right) \theta$, the resonance frequencies $\omega_{i}(i=1,2,3)$ of the considered metasurface depend on all the intrinsic parameters, i.e. the initial angle of rotation $\theta_{0}$, the mass ratio $\alpha_{m}$, the inertial moment $\alpha$, the normalized shear stiffness $K_{s}$ and the bending stiffness $K_{\theta}$. Using realistic materials studied earlier in [49], it is assumed here that the normalized shear and bending stiffnesses are both less than 0.1 and lie at the same value, i.e. $K_{s}=K_{\theta} \leq 0.1$. Additionally, the initial angle of rotation $\theta_{0}$ is set smaller than $30^{\circ}$. In Sections III and IV, the focus is placed on the case of homogeneous squares, i.e. $\alpha=1 / 3$, though other types of rotating elements with different inertial moments are considered in Section V.

In a previous theoretical study of a lumped-element, dual-resonance elastic metasurface model [42], it was demonstrated that to conduct the optimal frequency conversion from fundamental wave to second harmonic through the reflection process, a ratio of 2 between the two linear resonance frequencies of the metasurface is needed. The targeted conversion takes place with an excitation at the first resonance frequency. Regarding the current metasurface design with three degrees of freedom (rotation and translation of the squares, plus translation of the front plate), three resonance frequencies are involved $\omega_{i}(i=1,2,3)$ with $\omega_{1}<\omega_{2}<\omega_{3}$. Since the (geometric) nonlinearity is primarily excited by the rotation of squares, the excitation frequency $\omega$ should coincide with the resonance frequency, denoted as $\omega_{\theta}$, which corresponds to a rotation-dominated mode. Moreover, one of the other resonance frequencies, denoted here as $\omega_{u}$, should match $2 \omega_{\theta}$, in order to approximate the optimal conversion efficiency.

To satisfy the condition $\omega_{u}=2 \omega_{\theta}$, the mass ratio $\alpha_{m}$ can be determined in the linear and weakly dissipative case (see Fig. 2(b)). By simultaneously varying stiffnesses $\left(K_{s}, K_{\theta}\right)$ and initial angle of rotation $\theta_{0}$ in their considered intervals, the eigenmodes can be characterized by the magnitude of the ratio $\theta / U_{1}$ taken at the different resonance frequencies. A ratio $\theta / U_{1}$ with a magnitude greater than unity indicates a rotation-dominated mode, whereas a translation-dominated mode occurs with a ratio less than unity. Fig. 2(c) and Fig. 2(d) illustrate this ratio at resonance frequencies $\omega_{\theta}$ and $\omega_{u}$, respectively. It has been verified that within the considered range of metasurface parameters, the absolute ratio $\theta / U_{1}$ is maintained above 3 at $\omega_{\theta}$ and below 0.5 at $\omega_{u}$, i.e., $\omega_{\theta}$ (respectively $\omega_{u}$ ) corresponds to a rotation (respectively translation)-dominated mode.

However, once the displacement ratio $\theta / U_{1}$ deviates from unity (with an absolute value becoming much

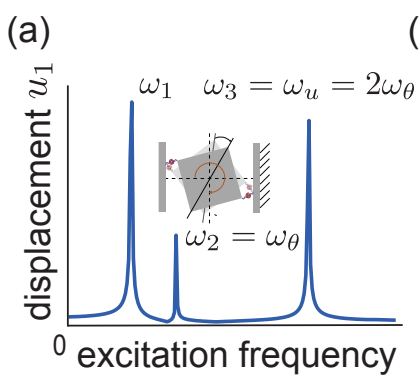

(b)
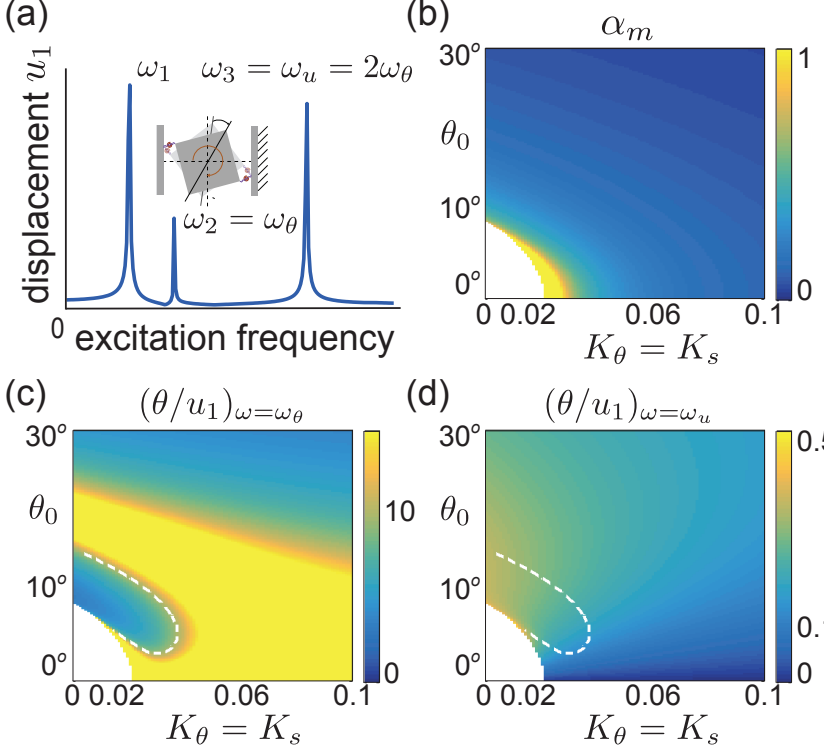

(d)

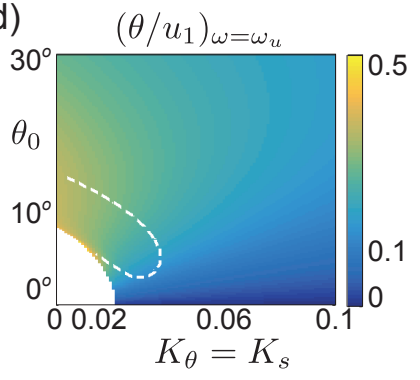

Figure 2. Eigenfrequencies and eigenvectors of the considered metasurface. In the linear dissipative regime, three resonance frequencies $\omega_{i}(i=1,2,3)$ are presented (a), related to either a translation-dominated movement, denoted $\omega_{u}$, or a rotationdominated movement denoted $\omega_{\theta}$, or a combination of both. When the resonance frequency condition $\omega_{u}=2 \omega_{\theta}$ is satisfied, the mass ratio $\alpha_{m}$ is determined for different values of initial angles of rotation $\theta_{0}$ and stiffnesses $K_{s}$ and $K_{\theta}(\mathrm{b})$. The ratio of $\theta$ to $U_{1}$ is examined as a function of $\theta_{0}$ and $K_{s}=K_{\theta}$ as well, at resonance frequencies $\omega_{\theta}$ and $\omega_{u}$ in (c) and (d), respectively. The optimal value range of stiffness and initial angle of rotation is indicated by the white dotted line in both (c) and (d).

smaller or much larger than unity), the rotation motion and translation motion turn out to be weakly coupled; consequently, the energy transfer from fundamental harmonic to higher harmonics becomes inefficient during the reflection process. In order to excite the rotation mode of the metasurface as much as possible while enhancing the intended nonlinear conversion, the ranges of stiffness and initial angle of rotation are limited, thus allowing for an absolute ratio $\theta / U_{1}$ less than 10 at frequency $\omega_{\theta}$ and greater than 0.1 at frequency $\omega_{u}$. Among the chosen displacement ratio threshold values, the optimal range of stiffness and initial angle of rotation values can be obtained, i.e. defined as $K_{s}=K_{\theta} \in(0,0.04)$ and $\theta_{0} \in\left(3^{\circ}, 15^{\circ}\right)$, as enclosed by the white dotted line in Fig. 2(c) and Fig. 2(d). The discussion in Section V verifies that the above choice of parameter space is indeed realistic and yields a high efficiency for the desired nonlinear conversion.

For the study of the nonlinear case in the following section IV, both the stiffness and initial angle of rotation of the metasurface are set as $K_{s}=K_{\theta}=0.02$ and $\theta_{0}=10^{\circ}$, which corresponds approximately to the center of the optimal parameter space region. The corresponding resonance frequencies in the linear regime, as normal- 
ized by $\omega_{0}$, are respectively $\Omega_{1}=0.7145, \Omega_{2}=1.0858$, and $\Omega_{3}=2.1716$. However, we found that as the incident amplitude increases, resulting in the activation of nonlinear effects, the metasurface resonance frequencies start shifting relative to the linear frequencies. This resonance shift will be considered in the following Section IV.

\section{NONLINEAR REFLECTION BY THE DESIGNED METASURFACE: OPTIMAL FREQUENCY CONVERSION}

Let's now consider a monochromatic source $f_{\text {inc }}(\Omega, \tau)=A_{\text {inc }} \cos (\Omega \tau)$ in order to solve semianalytically the considered problem [Eq. (3)] comprising nonlinear terms in the form of sine and cosine functions. An expansion up to the fourth order of all sinusoidal terms of [Eq. (3)] is firstly applied:

$$
\left\{\begin{aligned}
\cos \left(\theta_{0}+\theta\right) \approx & \cos \theta_{0}-\sin \theta_{0} \theta-\frac{1}{2} \cos \theta_{0} \theta^{2} \\
& +\frac{1}{6} \sin \theta_{0} \theta^{3}+\frac{1}{24} \cos \theta_{0} \theta^{4} \\
\sin \left(\theta_{0}+\theta\right) \approx & \sin \theta_{0}+\cos \theta_{0} \theta-\frac{1}{2} \sin \theta_{0} \theta^{2} \\
& -\frac{1}{6} \cos \theta_{0} \theta^{3}+\frac{1}{24} \sin \theta_{0} \theta^{4}
\end{aligned}\right.
$$

In the present study, which deals with the case of dynamic angles comparable to the initial angle of rotation, the considered expansion is determined to be sufficient since it yields reflection coefficient results with an accuracy to within 0.01 when compared to the numerical integration of the full problem described further below. The system of equations approximated by a polynomial form can now be solved using the Harmonic Balance Method (HBM) [50].

According to HBM, the solution $\{\mathbf{q}\}=\left\{\mathbf{U}_{\mathbf{1}}, \mathbf{U}_{\mathbf{2}}, \theta\right\}^{T}$ is developed as the sum of all generated harmonics:

$$
\{\mathbf{q}\}=\left\{\mathbf{q}_{\mathbf{0}}\right\}+\sum_{n=1}^{N}\left[\left\{\mathbf{C}_{\mathbf{n}}\right\} \cos (n \Omega \tau)+\left\{\mathbf{S}_{\mathbf{n}}\right\} \sin (n \Omega \tau)\right]
$$

with $\left\{\mathbf{q}_{\mathbf{0}}\right\}=\left\{\mathbf{C}_{\mathbf{0}}^{\mathbf{1}}, \mathbf{C}_{\mathbf{0}}^{\mathbf{2}}, \mathbf{C}_{\mathbf{0}}^{\mathbf{3}}\right\}^{T}$ indicating the constant terms of variables $U_{1}, U_{2}$ and $\theta,\left\{\mathbf{C}_{\mathbf{n}}\right\}$ and $\left\{\mathbf{S}_{\mathbf{n}}\right\}$ grouping respectively the magnitudes of cosine and sine terms of the three variables. $N$ denotes the finite number of harmonics under consideration, which is set at $N=10$, thus corresponding to a relative error of less than $10^{-15}$, compared to the solution for $N=9$. According to the vectorial form of the solution containing all considered $\mathrm{N}$ harmonics, the equation system [Eq. (3)] that is already approximated into a polynomial form, can be then rewritten in a matrix form that enables finally the numerical solution by applying the classical Newton-Raphson method. Once displacement $U_{1}$ is determined, the complex reflection coefficient of the n-th harmonic frequency component can be deduced as:

$$
\widetilde{R}_{n}=\delta_{n 1}+i \gamma n \Omega\left(\mathbf{C}_{n}^{1}-i \mathbf{S}_{n}^{1}\right),
$$

where $\delta_{n 1}$ is the Delta function, which always equals zero except when $n=1$. $\mathbf{C}_{n}^{1}$ and $\mathbf{S}_{n}^{1}$ denote the magnitudes of sinusoidal terms $\cos n \Omega \tau$ and $\sin n \Omega \tau$ of displacement $U_{1}$. The reflection coefficients obtained by HBM are considered as theoretical results and will be compared with the time domain simulation results at the end of the current section.

When the incident amplitude is relatively weak, the reflection coefficients obtained by HBM are close to the linear analytical solution. For instance, an excitation of dimensionless magnitude $A_{\text {inc }}=10^{-7}$ leads to an absolute difference in the reflection coefficient of less than $0.01 \%$, compared to the linear analytical solution. Therefore, for the following discussion of nonlinear phenomena, the excitation magnitude range considered extends from $A_{\text {inc }}=10^{-7}$ to $A_{\text {inc }}=10^{-4}$, i.e. from the linear case to amplitudes 3 orders of magnitude greater.

As mentioned at the end of Section III, once the excitation level is significant, the nonlinear resonance frequencies of the metasurface shift relative to the linear frequencies. Consequently, taking into account excitation frequency detuning with respect to the linear resonance frequencies, becomes necessary for the considered input amplitude range. In addition to the intrinsic parameters of the metasurface that have already been defined in Section III, the nonlinear reflection also depends on the propagation medium. By choosing herein two different excitation amplitudes, i.e. a relatively weak one with magnitude $A_{\text {inc }}=5 \times 10^{-6}$ and a stronger one with $A_{\text {inc }}=5 \times 10^{-5}$, the nonlinear reflection is thus being investigated simultaneously as a function of both the excitation frequency detuning $\Delta \Omega$ (normalized by $\omega_{0}$ ) and the medium impedance parameter $\gamma$, as shown in Fig. 3.

Through the reflection process and depending on input intensity, the frequency conversion can be achieved for a specific impedance value and for appropriate frequency detuning (see Fig. 3). In the case of $A_{\text {inc }}=5 \times 10^{-6}$, by setting the impedance parameter at $\gamma=0.008$ and considering a very small frequency detuning of $\Delta \Omega=-10^{-4}$, a second harmonic reflection coefficient of $R_{2}=0.418$ along with a near-zero fundamental coefficient $R_{1}=$ 0.0024 are obtained. In comparison, as the source amplitude increases to $A_{\text {inc }}=5 \times 10^{-5}$, the frequency detuning necessary to reduce reflection at the fundamental frequency becomes $\Delta \Omega=-1.7 \times 10^{-3}$, thus yielding a second harmonic reflection coefficient of $R_{2}=0.786$, accompanied by a fundamental coefficient $R_{1}=0.006$ at $\gamma=0.0195$. Furthermore, for the parameter ranges presented in Fig. 3, the reflection coefficients of harmonics higher than the second order are all found to be negligible compared to the second harmonic coefficient, with absolute values consistently less than 0.001 ; hence, these values will not be discussed any further in the present work.

The magnitude of excitation frequency detuning needed to minimize incident fundamental wave reflection corresponds to the resonance frequency shift of the metasurface with respect to the linear resonance fre- 

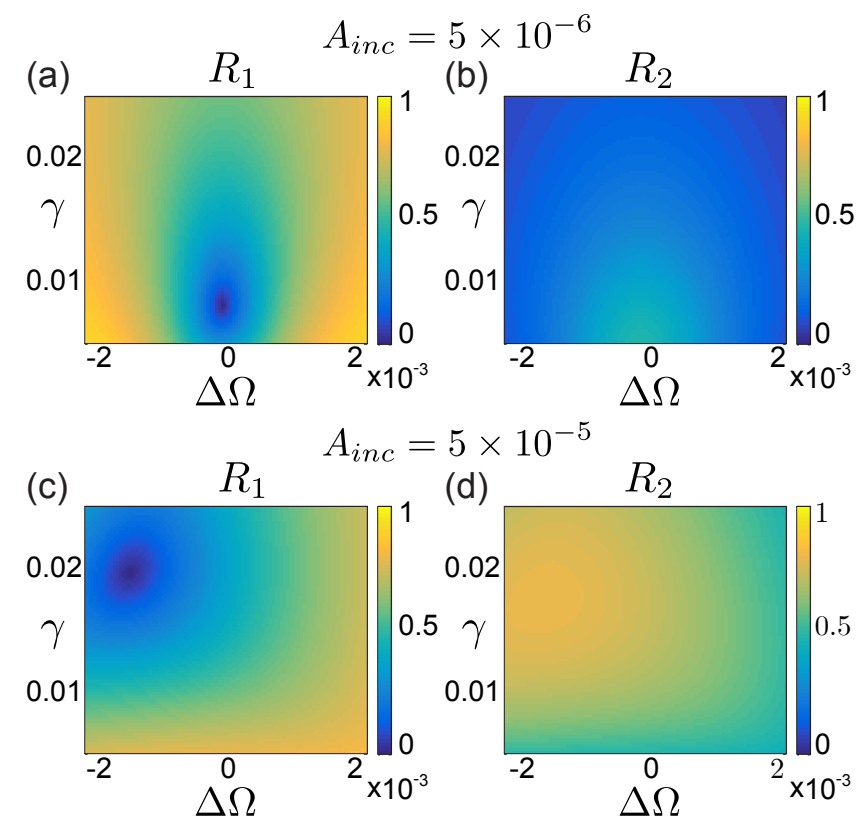

Figure 3. Absolute reflection coefficients of the fundamental and second harmonic components, denoted $R_{1}$ and $R_{2}$ respectively, as a function of both the dimensionless impedance parameter $\gamma$ and the normalized excitation frequency detuning $\Delta \Omega$. The latter is defined as the difference between the excitation frequency $\omega$ and the linear resonance frequency $\omega_{\theta}$, subsequently normalized by $\omega_{0}$, i.e., $\Delta \Omega=\left(\omega-\omega_{\theta}\right) / \omega_{0}$. When the input intensity is relatively weak, with a magnitude $A_{\text {inc }}=5 \times 10^{-6}$, the required excitation detuning is less $\left(\Delta \Omega=-1 \times 10^{-4}\right)$, as the maximum value of $R_{2}$ exceeds 0.4 (a) and (b). Whereas with a stronger source of magnitude $A_{\text {inc }}=5 \times 10^{-5}$, a frequency detuning of around $\Delta \Omega=-1.7 \times 10^{-3}$ is needed to totally absorb $R_{1}$, which does not alter the amplitude of the second harmonic $R_{2}$ to reach a maximum value of nearly 0.8 (c) and (d).

quency, under the considered level of excitation. More specifically, in order to analyze the resonance frequency shifts for the various source amplitudes indicated herein, i.e. weak level $A_{\text {inc }}=10^{-7}$ corresponding to the linear configuration and nonlinear levels $A_{\text {inc }}=5 \times 10^{-6}$ and $A_{\text {inc }}=5 \times 10^{-5}$, the metasurface kinetic energy at frequencies close to the linear resonance frequencies $\omega_{\theta}$ and $\omega_{u}$ has been introduced. For excitation around the rotation-dominated resonance frequency $\omega_{\theta}$, it has been verified that the maximum kinetic energy, which indicates the frequency position of the nonlinear resonance, actually shifts with increasing excitation amplitude, as illustrated in Fig. 4. Compared to the linear configuration defined by $A_{i n c}=10^{-7}$, the resonance shift remains negligible under a weak nonlinear level excitation with $A_{\text {inc }}=5 \times 10^{-6}$, while it becomes significant when the excitation level increases to $A_{\text {inc }}=5 \times 10^{-5}$. The frequency shift between the nonlinear and linear resonance frequencies coincides exactly with the optimal excitation detuning, as introduced previously in Fig. 3, in order to minimize reflection of the fundamental wave. (a)

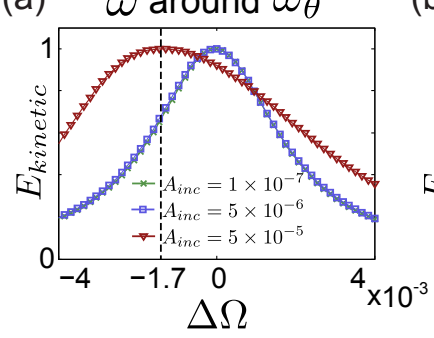

(b) $\omega$ around $\omega_{u}$

Figure 4. Kinetic energy $E_{\text {kinetic }}$ of the metasurface at various excitation levels, from a linear configuration with $A_{\text {inc }}=10^{-7}$ to a weakly nonlinear configuration with $A_{i n c}=5 \times 10^{-6}$ and a highly nonlinear configuration $A_{i n c}=5 \times 10^{-5}$, respectively, for the cases of: (a) excitation frequencies $\omega$ close to the linear rotation-dominated resonance frequency $\omega_{\theta}$, and (b) excitation frequencies $\omega$ close to the linear translation-dominated resonance frequency $\omega_{u}$ verifying $\omega_{u}=2 \omega_{\theta}$. During the kinetic energy test, the metasurface is excited, at each excitation frequency, by 1,000 periods of a sine signal. The dotted black line in (a) indicates the resonance shift under excitation $A_{\text {inc }}=5 \times 10^{-5}$, which corresponds exactly to the optimal excitation detuning introduced in Fig. 3.

Nevertheless, for excitation around $\omega_{u}$ corresponding to a translation-dominated motion (Fig. 4b), as opposed to excitation around $\omega_{\theta}$, the excitation level does not influence the kinetic energy curve. Hence, when the excitation frequency is detuned to compensate for the frequency shift of resonance $\omega_{\theta}$, the reflection of the fundamental wave can become minimized, whereas the second harmonic (which is detuned twice as fast as the fundamental harmonic) will barely change its reflection coefficient $R_{2}$. This result is due to the fact that the corresponding frequency detuning around resonance $\omega_{u}$ does not introduce as much of a variation in kinetic energy as the detuning around $\omega_{\theta}$. Accordingly, the excitation detuning simultaneously enables minimizing the reflection of the fundamental wave while maintaining the nonlinear conversion efficiency into the reflected second harmonic wave.

In Fig. 5, the evolution of both the fundamental and second harmonic reflection coefficients are examined over the gradual increase in excitation amplitude, from the linear case to the case enabling activation of nonlinear effects. The comparison between cases, whether or not excitation detuning has been taken into consideration, is presented as well. These findings serve to confirm that the excitation detuning primarily affects the fundamental wave reflection and much less so the conversion towards second harmonic frequency.

When excitation detuning is not introduced (the source $f_{\text {inc }}=A_{\text {inc }} \cos \omega t$ at frequency $\omega=\omega_{\theta}$ coincides with the linear rotation-dominated resonance), the reflection coefficients $R_{1}$ and $R_{2}$ are plotted in Fig. 5 for an excitation amplitude range starting from the linear configuration $A_{i n c}=10^{-7}$. With an increasing excitation amplitude, due to the introduced frequency matching, 

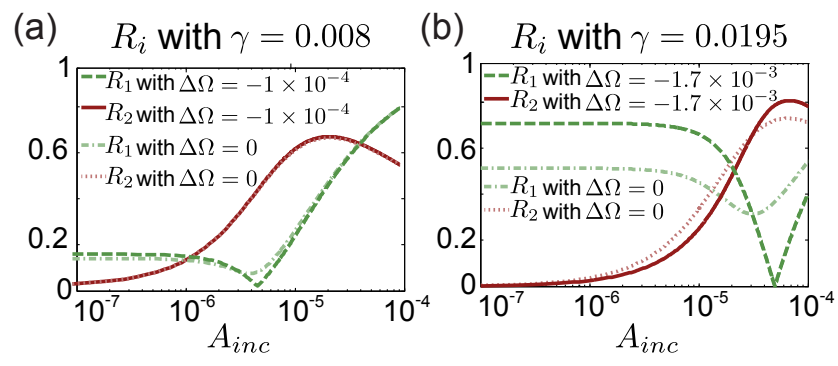

Figure 5. Absolute reflection coefficient of fundamental $\left(R_{1}\right)$ and second harmonics $\left(R_{2}\right)$, as investigated by varying the excitation amplitude from a linear level $\left(A_{\text {inc }}=10^{-7}\right)$ to a nonlinear level $\left(A_{\text {inc }}=10^{-4}\right)$. Frequency detuning is introduced in order to eliminate reflection of the fundamental wave at the desired excitation amplitude, such that (a) $A_{i n c}=5 \times 10^{-6}$ and (b) $A_{\text {inc }}=5 \times 10^{-5}$, with the impedance parameter defined as $\gamma=0.008$ and $\gamma=0.0195$, respectively.

i.e., $2 \omega=2 \omega_{\theta}=\omega_{u}$, the quadratic nonlinear effect is significantly amplified and appears first, thus yielding an efficient growth of $R_{2}$.

When the source amplitude is further increased, cubic nonlinear effects start to appear, stemming from both the cubic nonlinear terms of the expansions in the full problem (3) and the nonlinear cascade process (next-order interaction) from the quadratic terms. Cubic nonlinear effects induce a variation of $R_{1}$ via self-action on $\omega$ while the quadratic via interactions between $\omega$ and $2 \omega$, respectively. Given the result of excitation matching $\omega=\omega_{\theta}$, the nonlinear part of $R_{1}$ is magnified, yielding a clear increase in $R_{1}$ following an initial decrease, as shown in Fig. 5. However, the third harmonic component, which is also generated due to the nonlinear effect yet mismatched with the system resonances, remains negligible with a magnitude of less than 0.001 .

By taking advantage of excitation detuning, the extreme value of $R_{1}$, occurring due to the activation of cubic nonlinear effects, can thus be minimized or even eliminated under a specific impedance parameter value $\gamma$. Nevertheless, since the higher resonance $\omega_{u}$ is less sensitive to excitation detuning than the rotation-dominated resonance $\omega_{\theta}$, the second harmonic reflection coefficient $R_{2}$ is not influenced to the same extent as $R_{1}$ for the fundamental wave. For excitation level $A_{i n c}=5 \times 10^{-6}$, by introducing frequency detuning $\Delta \Omega=-1 \times 10^{-4}$ and setting $\gamma=0.008$, the fundamental wave reflection coefficient is minimized to $R_{1}=0.0024$, while the second harmonic can reach a reflection coefficient of $R_{2}=0.418$, as shown in Fig. 5(a). In contrast, a frequency detuning of $\Delta \Omega=-1.7 \times 10^{-3}$ enables $R_{1}=0.006$ at excitation level $A_{\text {inc }}=5 \times 10^{-5}$ under $\gamma=0.195$, along with a second harmonic reflection coefficient as high as $R_{2}=0.786$, see Fig. 5(b). Note that these parameters are the same as those used in Fig. 3. In terms of energy, the two above configurations correspond to around $17 \%$ and $62 \%$ of energy concentrated on the second harmonic under excitation magnitudes $A_{\text {inc }}=5 \times 10^{-6}$ and $A_{\text {inc }}=5 \times 10^{-5}$
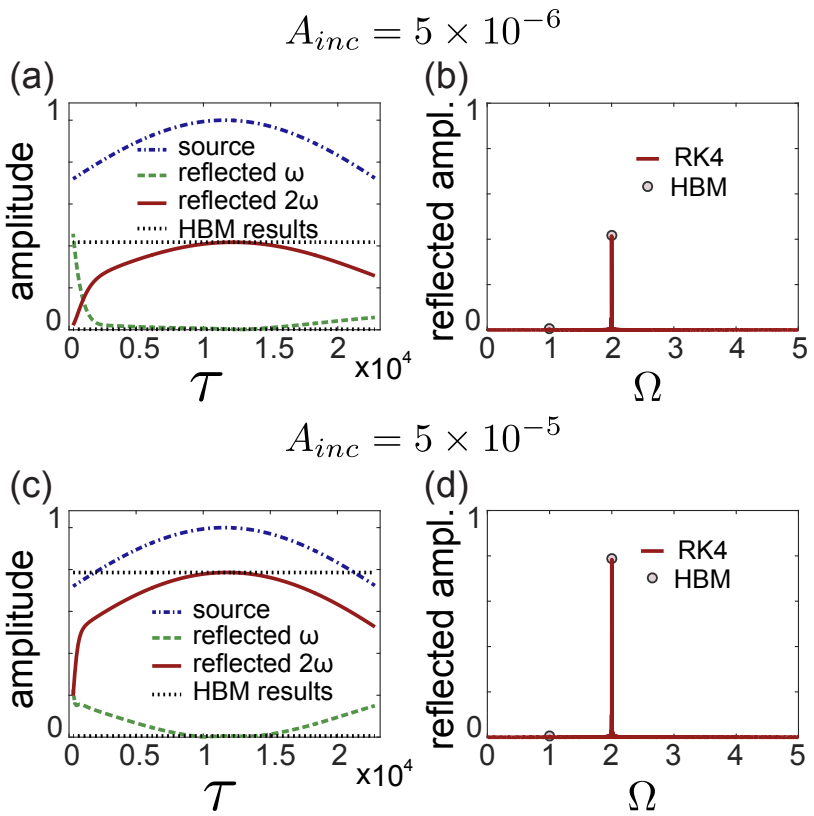

(d)

Figure 6. Theoretical and numerical results for the nonlinear metasurface reflection, as obtained with the Harmonic Balance Method (HBM) and the fourth-order Runge-Kutta (RK4) method, respectively, for the case of a relatively weak excitation with amplitude $A_{i n c}=5 \times 10^{-6}$ (a) and (b), and for the case of a stronger excitation with amplitude $A_{\text {inc }}=5 \times 0^{-5}$ (c) and (d). The frequency axes are normalized by the detuned excitation frequency. By considering a wave packet source with characteristic width $N_{T}=4000 T$, the RK4 results are compared to the theoretical HBM results. Magnitudes of Short-Term Fourier Transforms taken at fundamental and second harmonic frequencies, by showing the temporal variation in the reflected wave spectrum (a) and (c), and by exploring the Fourier Transform of the central 1,000 periods of the reflected temporal wave (b) and (d), respectively.

respectively, both with less than $0.01 \%$ energy reflected in the fundamental wave.

Since an analytical solution does not exist for the full nonlinear problem (HBM is applied to the problem approximated by a polynomial expansion of all the nonlinear terms), numerical solutions to the full nonlinear problem with HBM results can now be compared. System Eq. (3) is solved numerically using the classical fourth-order Runge-Kutta (RK4) integration method $[?]$, and the excitation is a Gaussian-modulated wave packet source of the form,

$$
\sigma_{i n c}(\Omega, \tau)=A_{i n c} f_{i n c}(\Omega, \tau)=A_{i n c} \sin (\Omega \tau) e^{-\frac{\left(\tau-\tau_{0}\right)^{2}}{(\omega T)^{2}}}
$$

with $\tau=\omega t, T$ the characteristic temporal width of the wave packet, and $\tau_{0}$ the dimensionless time center of the packet. The reflected wave signal is obtained with the help of relation Eq. (2) once the temporal displacement $u_{1}$ of the front plate has been determined.

The time-frequency analysis of the reflected signals can therefore be performed using the spectrogram method, in 
yielding a reflected time-dependent spectrum at the fundamental and second harmonic frequencies, i.e. $\omega$ and $2 \omega$, as shown in Fig. 6(a) and Fig. 6(c), for the considered excitation amplitudes $5 \times 10^{-6}$ and $5 \times 10^{-5}$, respectively. The wave packet source has a characteristic half-height duration of 4000 periods. It has been demonstrated that the RK4 simulation reaches the theoretical results of HBM when the excitation amplitude lies close to the maximum magnitude $A_{\text {inc }}$. Fig. 6(b) and Fig. 6(d) present the comparison between HBM results and RK4 simulations when the signal spectrum is computed over the 1000 center periods of the reflected temporal signal. A good level of agreement is observed between the two methods for both plotted excitation amplitudes, each with an absolute difference of less than 0.01 .

\section{DISCUSSION}

According to the proposed metasurface design, which comprises rotating squares, the desired frequency conversion from the incoming fundamental wave to the reflected second harmonic can in fact be achieved. In addition to the specific design presented above in Section IV (with fixed intrinsic parameters such as inertial moment $\alpha=1 / 3$, shear and bending stiffnesses $K_{s}=K_{\theta}=0.02$ and initial angle of rotation $\theta_{0}=10^{\circ}$ ), it is possible to explore an even wider parameter space, offering greater tunability opportunities.

With an initial angle of rotation set at $\theta_{0}=10^{\circ}$ and $\theta_{0}=20^{\circ}$, respectively, and for stiffness in the range $K_{s}=K_{\theta} \in(0,0.1)$, the optimal conversion is sought by varying the impedance parameter $\gamma$ and introducing the excitation frequency shift $\Delta \Omega$. The optimal results of reflection coefficient $R_{2}$ as a function of stiffness $K_{s}=K_{\theta}$ are shown in Fig. 7(a). Note that the impedance parameter $\gamma=\rho c S / m \omega_{0}$ can be modified by changing the size or the mass of the squares, which allows for impedance tuning. The final parameter of the metasurface is the mass ratio between front plate and rotating squares, denoted $\alpha_{m}$. This parameter is defined to be positive and such that it satisfies the necessary frequency condition $\omega_{u}=2 \omega_{\theta}$ in the linear regime.

A similar analysis has been repeated for stiffnesses set at $K_{s}=K_{\theta}=0.02$ and $K_{s}=K_{\theta}=0.06$, respectively. The maximum second harmonic reflection coefficient has been estimated for initial angles of rotation $\theta_{0} \in\left(0^{\circ}, 30^{\circ}\right)$, as illustrated in Fig. 7(b). The excitation level is set at $A_{\text {inc }}=5 \times 10^{-5}$ for both parametric studies conducted in Fig. 7(a) and Fig. 7(b). It is shown that in the case of rotating square masses, i.e. $\alpha=1 / 3$, the generation of a second harmonic remains significant over the entire optimal value ranges for stiffness $K_{s}=K_{\theta} \in(0,0.04)$ and initial angles of rotation $\theta_{0} \in\left(3^{\circ}, 15^{\circ}\right)$, as enclosed by the white dotted lines in Fig. 2 of Section III. Within this optimal parameter range, according to the results in Fig. $7, R_{2}$ is observed (a)

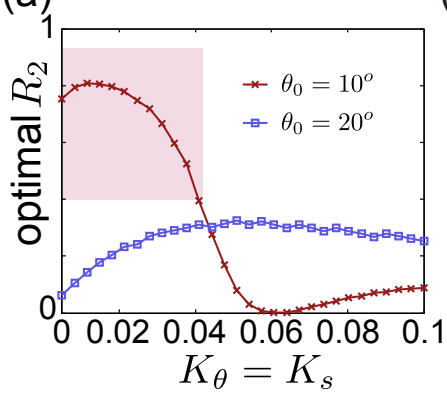

(b)

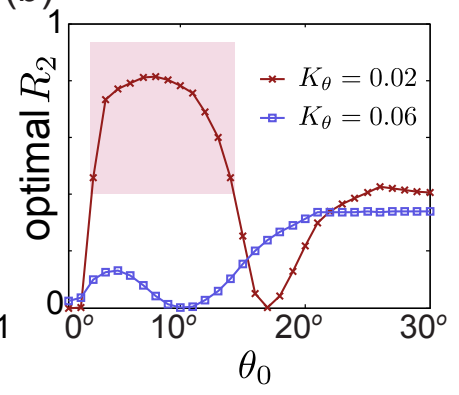

Figure 7. Optimal frequency conversion effect achieved for various physical properties of the proposed metasurface, under an excitation level of $A_{\text {inc }}=5 \times 10^{-5}$. The maximum absolute value of the reflection coefficient of the second harmonic $R_{2}$ is identified by varying the impedance parameter and excitation frequency detuning simultaneously, for stiffnesses in the range $K_{s}=K_{\theta} \in(0,0.1)$ and an initial angle of rotation set at $10^{\circ}$ and $20^{\circ}$ in (a), and for an initial angle of rotation in the range $\theta_{0} \in\left(0^{\circ}, 30^{\circ}\right)$ and stiffnesses set at 0.02 and 0.06 in (b). Shaded zones indicate the optimal ranges of initial angle of rotation and stiffnesses, i.e. $K_{s}=K_{\theta} \in(0,0.04)$ and $\theta_{0} \in\left(3^{\circ}, 15^{\circ}\right)$, thus yielding an efficient second harmonic reflection with $R_{2}$ greater than 0.4 . The results presented have been output by HBM.

to be greater than 0.4 , while $R_{1}$ remains less than 0.05 .

Furthermore, the desired nonlinear phenomena can be derived for various rotating unit shapes, as characterized by different inertial moments $\alpha$. Let's recall herein that a point mass corresponds to a zero inertial moment $\alpha=0$, whereas a hollow square (the entire mass distributed at the edges) has an inertial moment of $\alpha=2 / 3$. An inertial moment $\alpha \in[0.02,0.66]$ is thus considered (although in theory this moment could be removed from the value range by, for example, using gyroscopes in the design). Within the considered range of inertial moment and for the sake of computational efficiency, the initial angle of rotation has been set at $10^{\circ}$ and $8^{\circ}$, respectively, as these values are found to be favorable for generating the desired reflection over the entire range of inertial moment. The maximum generation of reflected second harmonic along with the minimum fundamental reflected wave can be determined by simultaneously varying all other intrinsic metasurface parameters, i.e. stiffness $K_{s}=K_{\theta}$, impedance parameter $\gamma$ and required excitation detuning $\Delta \Omega$. Like in the previous study presented in Fig. 7 , the last parameter $\alpha_{m}$, i.e. the mass ratio, is chosen so that the necessary condition $\omega_{u}=2 \omega_{\theta}$ is satisfied.

According to the results shown in Fig. $8, R_{2}$ is always greater than 0.4 over the entire range of inertial moment $\alpha$ and possibly greater than 0.74 for inertial moment in the range $\alpha \in(0.2,0.66)$, the energy distribution on the second harmonic is thus remained within the range $(16 \%, 55 \%)$. Hence, not only can the proposed design composed of homogeneous squares having an inertial moment $\alpha=1 / 3$ produce the desired nonlinear frequency conversion, but other rotating periodic struc- 


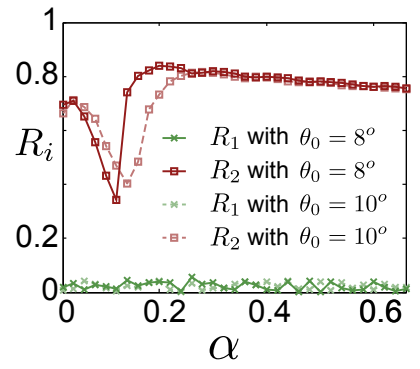

Figure 8. Optimal frequency conversion effect achieved for various metasurface unit cell shapes, i.e. for different inertial moments $\alpha$ of rotating elements. The maximum conversion is determined as a function of $\alpha$ over the range of $[0.02,0.66]$ by varying the impedance parameter $\gamma$, excitation frequency detuning $\Delta \Omega$ and stiffness $K_{s}=K_{\theta}$ simultaneously. In order to lessen the calculation burden, the initial angle of rotation has been set at $10^{\circ}$ and $8^{\circ}$ respectively, as these values are found to be favorable for producing the desired reflection over the entire inertial moment range.

tures are also capable of efficiently generating the second harmonic through the reflection process, provided that the inertial moments of their unit cells are included in the above value range $\alpha \in(0.2,0.66)$. The possible metasurface rotating element shapes may, for instance: be square, rectangular or diamond-shaped; contain holes or additional masses; constitute the centrally symmetric four-corner structure such as a cross (the inertial moment $\alpha$ depends on the exact length-to-width ratio of each edge but is generally slightly less than $1 / 3$ ); or even extend to other novel shapes based on regular polygons or centro-symmetric structures.

\section{CONCLUSION}

In conclusion, through a realistic metasurface design inspired from recent results on the dynamics of soft architected rotating square structures [31, 49], the possibility of achieving near-perfect absorption of the incoming fundamental wave has been demonstrated herein, along with an efficient conversion into the second harmonic frequency. By introducing appropriate excitation frequency detuning, which compensates for the nonlinear frequency shift of one rotation-dominated resonance $\omega_{\theta}$ of the metasurface, the reflection of the incoming fundamental wave can be as low as $R_{1}<0.05$. Since the translationdominated resonance has a broader frequency response and a barely noticeable nonlinear frequency shift compared to the rotation-dominated resonance, the efficiency of the conversion towards the second harmonic frequency is much less influenced by the introduced frequency detuning. The corresponding reflection coefficient $R_{2}$ can be consistently maintained above 0.4 and even reach values exceeding 0.8 depending on the excitation level.

In order to validate the theoretical results obtained with HBM in considering a monochromatic source, the nonlinear reflection of a wave packet has also been examined by numerically integrating the system of fully nonlinear motion equations. An excellent level of agreement has been obtained between the theoretical results output by HBM and the implemented numerical results, provided the characteristic temporal width of the wave packet signal is large enough, in accordance with expectations. Moreover, the value ranges of intrinsic metasurface parameters that efficiently lead to the desired frequency conversion have also been determined, i.e. for the metasurface unit cell consisting of homogeneous rotating squares $(\alpha=1 / 3)$, the stiffness and initial angle of rotation in the range of $K_{s}=K_{\theta} \in(0,0.04)$ and $\theta_{0} \in\left(3^{\circ}, 15^{\circ}\right)$, respectively, thus allowing for efficient second harmonic generation with a reflection coefficient $R_{2}$ always greater than 0.4 .

The proposed metasurface design, which is capable of enhancing the nonlinear effect, has been found to be extendable to a series of designs with rotating unit cells, not only of a square shape but other available structures as well, possessing an inertial moment within the value range of $\alpha \in[0.02,0.66]$. The second harmonic reflection coefficient may indeed exceed 0.74 if $\alpha \in[0.2,0.66]$, corresponding to around $55 \%$ of energy concentrated on the second harmonic together with extremely small energy $(<0.01 \%)$ reflected in the fundamental wave. According to the present study and the previous work [42], one should notice that the achievement of the desired nonlinear conversion needs a matching between the intrinsic resonances of the considered meta-structure and the frequencies of the generated harmonics, which can be enabled by the appropriate metasurface design, i.e. structural parameters within specific value ranges. Furthermore, the proposed family of metasurface made of rotating unit cells can also provide other types of nonlinearity, depending on the choice of the structural parameters. The proposed metasurface design rules apply not only to the presented second harmonic enhancement, but could also optimize other types of nonlinear effects, such as the third harmonic generation.

Nevertheless, the reported acoustic / elastic wave control by the scattering process is limited herein to reflection at normal incidence. Consequently, the considered nonlinear conversion would need to be investigated in other configurations, such as transmission by a thin and resonant meta-interface. The presented types of designs with rotating units, given the possibility of managing their dispersive and nonlinear elastic properties, open avenues for enhancing nonlinear wave control. By considering a larger number of layers with varying properties, the rather rudimentary scattering process studied herein could potentially be extended to broader operating frequency ranges as well as to other nonlinear processes. Moreover, such nonlinear wave scattering properties could become useful for applications in wave pulse mitigation, acoustic diode design and nonreciprocal transmission systems. 


\section{ACKNOWLEDGMENTS}

The authors hereby acknowledge the support of the "Le Mans Acoustique HUB" and PROPASYM projects funded by France's Loire Valley Region (Région Pays-dela-Loire).
[1] A. Santillán and S. I. Bozhevolnyi, Phys. Rev. B 84, 064304 (2011).

[2] G. Theocharis, O. Richoux, V. R. García, A. Merkel, and V. Tournat, New J. Phys 16, 093017 (2014).

[3] A. Santillán and S. I. Bozhevolnyi, Phys. Rev. B 89, 184301 (2014).

[4] V. M. Agranovich, Y. R. Shen, R. H. Baughman, and A. A. Zakhidov, Phys. Rev. B 69, 165112 (2004).

[5] G. V. Eleftheriades and K. G. Balmain, NegativeRefraction Metamaterials: Fundamental Principles and Applications (Wiley IEEE Press, 2005).

[6] J. Yao, Z. Liu, Y. Liu, Y. Wang, C. Sun, G. Bartal, A. M. Stacy, and X. Zhang, Science 321, 930 (2008).

[7] J. Li and C. T. Chan, Phys. Rev. E 70, 055602 (2004).

[8] D. R. Smith, J. B. Pendry, and M. C. K. Wiltshire, Sci. 305, 788 (2004).

[9] J. Christensen, Z. Liang, and M. Willatzen, Phys. Rev. B 88, 100301 (2013).

[10] N. Kaina, F. Lemoult, M. Fink, and G. Lerosey, Nature 525, 77 (2015).

[11] T. Devaux, V. Tournat, O. Richoux, and V. Pagneux, Phys. Rev. Lett. 115, 234301 (2015).

[12] F. Lemoult, N. Kaina, M. Fink, and G. Lerosey, Nature Physics 9, 55 (2012).

[13] V. Romero-García, G. Theocharis, O. Richoux, A. Merkel, V. Tournat, and V. Pagneux, Sci. Rep. 6, srep19519 (2016).

[14] X. Jiang, B. Liang, R. Li, X. Zou, L. Yin, and J. Cheng, Appl. Phys. Lett. 105, 243505 (2014).

[15] M. Yang, G. Ma, Z. Yang, and P. Sheng, EPJ Appl. Metamat. 2, 10 (2015).

[16] J. Mei, G. Ma, M. Yang, Z. Yang, W. Wen, and P. Sheng, Nat. Commun. 3, ncomms1758 (2012).

[17] Y. Li and M. A. Badreddine, Appl. Phys. Lett. 108, 063502 (2016).

[18] M. Yang, C. Meng, C. Fu, Y. Li, Z. Yang, and P. Sheng, Appl. Phys. Lett. 107, 104104 (2015).

[19] Y. Duan, J. Luo, G. Wang, Z. H. Hang, B. Hou, J. Li, P. Sheng, and Y. Lai, Sci. Rep. 5, srep12139 (2015).

[20] P. Sheng, J. Acoust. Soc. Am. 141, 3575 (2017).

[21] W. Cai, U. K. Chettiar, A. V. Kildishev, and V. M. Shalaev, Nat. Photonics. 1, 224 (2007).

[22] J. Valentine, J. Li, T. Zentgraf, G. Bartal, and X. Zhang, Nat. Mater. 8, 568 (2009).

[23] G. Zhu, J. Appl. Phys. 113, 163103 (2013).

[24] L. Zigoneanu, B.-I. Popa, and S. A. Cummer, Nature Materials 13, 352 (2014).

[25] N. Boechler, G. Theocharis, and C. Daraio, Nat. Mater. 10, 665 (2011).

[26] R.-Q. Li, B. Liang, Y. Li, W.-W. Kan, X.-Y. Zou, and J.-C. Cheng, Appl. Phys. Lett. 101, 263502 (2012).

[27] J.-S. Chen, I.-L. Chang, W.-T. Huang, L.-W. Chen, and G.-H. Huang, AIP Adv. 6, 095020 (2016).

[28] J. Christensen and F. J. G. de Abajo, Phys. Rev. Lett. 108, 124301 (2012).

[29] J. Cabaret, P. Béquin, G. Theocharis, V. Andreev, V. E.
Gusev, and V. Tournat, Phys. Rev. Lett. 115, 054301 (2015).

[30] C. Daraio, V. F. Nesterenko, E. B. Herbold, and S. Jin, Phys. Rev. E 73, 026610 (2006).

[31] B. Deng, J. R. Raney, V. Tournat, and K. Bertoldi, Phys. Rev. Lett. 118, 204102 (2017).

[32] V. Grubsky and A. Savchenko, Opt. Express 13, 6798 (2005).

[33] S. Sun, N. Yi, W. Yao, Q. Song, and S. Xiao, Opt. Express 22, 26613 (2014).

[34] L. Wang, J.-H. Zhang, Z.-Q. Wang, C. Liu, M. Li, F.-H. Qi, and R. Guo, Phys. Rev. E 93, 012214 (2016).

[35] R. A. Van Gorder, Phys. Rev. D 95, 096007 (2017).

[36] J. Lee, M. Tymchenko, C. Argyropoulos, P.-Y. Chen, F. Lu, F. Demmerle, G. Boehm, M.-C. Amann, A. Al, and M. A. Belkin, Nature 511, 65 (2014).

[37] P.-Y. Chen, C. Argyropoulos, G. D'Aguanno, and A. Al, ACS Photonics 2, 1000 (2015).

[38] R. Chandrasekar, N. K. Emani, A. Lagutchev, V. M. Shalaev, C. Ciracì, D. R. Smith, and A. V. Kildishev, Opt. Mater. Express 5, 2682 (2015).

[39] Stephanie C. Konarski, Michael R. Haberman, and Mark F. Hamilton, J. Acoust. Soc. Am. 144, 3022-3035 (2018).

[40] F. Allein, V. Tournat, V. E. Gusev, and G. Theocharis, Applied Physics Letters 108, 161903 (2016), https://doi.org/10.1063/1.4947192.

[41] J. Cabaret, V. Tournat, and P. Béquin, Phys. Rev. E 86, 041305 (2012).

[42] X. Guo, V. E. Gusev, K. Bertoldi, and V. Tournat, Journal of Applied Physics 123, 124901 (2018), https://doi.org/10.1063/1.5015952.

[43] M. I. Hussein, M. J. Leamy, and M. Ruzzene, Applied Mechanics Reviews 66, 040802 (2014).

[44] S. Shan, S. H. Kang, J. R. Raney, P. Wang, L. Fang, F. Candido, J. A. Lewis, and K. Bertoldi, Adv. Mater. 27, 4296 (2015).

[45] B. Florijn, C. Coulais, and M. van Hecke, Phys. Rev. Lett. 113, 175503 (2014).

[46] K. Bertoldi and M. C. Boyce, Phys. Rev. B 77, 052105 (2008).

[47] P. Wang, F. Casadei, S. Shan, J. C. Weaver, and K. Bertoldi, Phys. Rev. Lett. 113, 014301 (2014).

[48] O. R. Bilal, A. Foehr, and C. Daraio, Advanced Materials 29, 1700628 (2017).

[49] B. Deng, P. Wang, Q. He, V. Tournat, and K. Bertoldi, Nature Communications 9, 3410 (2018).

[50] V. Marinca and N. Herisanu, "The method of harmonic balance," in Nonlinear Dynamical Systems in Engineering: Some Approximate Approaches (Springer Berlin Heidelberg, Berlin, Heidelberg, 2011) pp. 31-45E. Hairer, S. P. Norsett, and G. Wanner, "Runge-kutta and extrapolation methods," in Solving Ordinary Differential Equations I: Nonstiff Problems (Springer Berlin Heidelberg, Berlin, Heidelberg, 1993) pp. 129-353. 